\title{
Measurements and simulations of muon-induced neutrons
}

\author{
H. M. Araújo ${ }^{a, b}$, J. Blockley ${ }^{c}$, C. Bungau ${ }^{b, a}$, M. J. Carson ${ }^{c}$, H. Chagani ${ }^{c}$, E. Daw ${ }^{c}$, \\ B. Edwards ${ }^{a, b}$, C. Ghag $^{d}$, E. V. Korolkova ${ }^{c}$, V. A. Kudryavtsev ${ }^{c}{ }^{*}$ P. K. Lightfoot ${ }^{c}$, \\ A. Lindote ${ }^{e}$, I. Liubarsky ${ }^{b, a}$, R. Lüscher ${ }^{b}$, P. Majewski ${ }^{c}$, K. Mavrokoridis ${ }^{c}$, \\ J. E. McMillan ${ }^{c}$, A. St. J. Murphy ${ }^{d}$, S. M. Paling ${ }^{c}$, J. Pinto da Cunha ${ }^{e}$, R. M. Preece ${ }^{b}$, \\ M. Robinson ${ }^{c}$, N. J. T. Smith ${ }^{b}$, P. F. Smith ${ }^{b}$, N. J. C. Spooner ${ }^{c}$, T. J. Sumner ${ }^{a}$, \\ R. J. Walker ${ }^{a}$, H. Wang ${ }^{f}$ and J. White ${ }^{g}$ \\ ${ }^{a}$ Blackett Laboratory, Imperial College London, UK \\ ${ }^{b}$ Particle Physics Department, STFC Rutherford Appleton Laboratory, UK \\ ${ }^{c}$ Department of Physics \& Astronomy, University of Sheffield, UK \\ ${ }^{d}$ School of Physics, University of Edinburgh, UK \\ ${ }^{e}$ LIP-Coimbra \& Department of Physics of the University of Coimbra, Portugal \\ ${ }^{f}$ Department of Physics \& Astronomy, University of California, Los Angeles, USA \\ ${ }^{g}$ Department of Physics, Texas A\&M University, USA
}

\begin{abstract}
The first measurements of the muon-induced neutron flux at the Boulby Underground Laboratory are presented. The experiment was carried out with an 0.73 tonne liquid scintillation detector that also served as an anticoincidence system for the ZEPLIN-II direct dark matter search. The experimental method exploited the delayed coincidences between high-energy muon signals and gamma-rays from radiative neutron capture on hydrogen or other elements. The muon-induced neutron rate, defined as the average number of detected neutrons per detected muon, was measured as $0.079 \pm 0.003$ (stat.) neutrons/muon using neutron-capture signals above $0.55 \mathrm{MeV}$ in a time window of 40-190 $\mu$ s after the muon trigger. Accurate Monte Carlo simulations of the neutron production, transport and detection in a precisely modeled laboratory and experimental setup using the GEANT4 toolkit gave a result 1.8 times higher than the measured value. The difference greatly exceeds all statistical and systematic uncertainties. As the vast majority of neutrons detected in the current setup were produced in lead we evaluated from our measurements the neutron yield in lead as $(1.31 \pm 0.06) \times 10^{-3}$ neutrons $/$ muon $/\left(\mathrm{g} / \mathrm{cm}^{2}\right)$ for a mean muon energy of about $260 \mathrm{GeV}$.
\end{abstract}

Identification of dark matter 2008

August 18-22, 2008

Stockholm, Sweden

${ }^{*}$ Speaker and corresponding author; e-mail: v.kudryavtsev@ sheffield.ac.uk 


\section{Introduction}

Background from muon-induced neutrons is one of the most important limitations to detector sensitivity in underground experiments, in particular for direct dark matter searches. In WIMP dark matter detectors nuclear recoils of $\mathrm{keV}$ energies originating in neutron elastic scattering mimic WIMP-nucleus interactions. Neutrons are produced by muons via 4 main processes: i) negative muon capture (relevant only to stopping muons, or for shallow depths less than $100 \mathrm{~m} \mathrm{w}$. e.); ii) direct muon-induced spallation of a nucleus; iii) photoproduction of neutrons or photon-induced spallation (mainly in electromagnetic cascades initiated by muons); iv) hadroproduction of neutrons (mainly in hadronic or nuclear cascades originated by muons). The relative contribution of different processes in different models have been investigated in Refs. [1, 2]. In this paper we describe the measurement of the total neutron yield from cosmic-ray muons carried out at the Boulby Underground Laboratory (Boulby mine, North Yorkshire, UK). This is the first experiment with a large mass of lead as a target for which direct measurements and detailed Monte Carlo are compared directly enabling the test of the models.

\section{Detector, experiment and data analysis}

The measurements were carried out at the Boulby Underground Laboratory at a depth of about $1070 \mathrm{~m}$ or $2850 \mathrm{~m}$ w. e. The liquid scintillator of an active veto system working also in anticoincidence with the WIMP dark matter detector ZEPLIN-II [3] was used for muon and neutron detection. The veto detector is viewed by ten PMTs and is surrounded by a 'castle' made of lead and designed to shield the ZEPLIN-II dark matter detector from gamma-rays from rock. The total weight of lead is about 50 tonnes making it an excellent target for neutron production. All materials of and around the veto detector and their exact locations within the laboratory were put into the simulation code based on GEANT4 toolkit [4]. More details can be found in Refs. [5, 6].

Signals from the ten PMTs were passed through discriminators and a coincident unit. The unit generated a logic pulse if the amplitudes of the analogue pulses from three or more PMTs exceeded an average amplitude of one photoelectron within $150 \mathrm{~ns}$. The logic pulses were used in the off-line analysis to identify 'true' pulses and reject noise and other background pulses caused by radioactivity close to a single PMT (for instance on the PMT window) without coincidences with any other PMT. Analogue signals from all ten PMTs were summed together by an adder and the sum signal was fed into a waveform digitiser sampling at a rate of $500 \mathrm{MHz}$.

The principle of neutron detection was based on the delayed coincidences between the first, high-energy, pulse from a muon or muon-induced cascade and the delayed second, low-energy, pulse from neutron capture gamma(s). In the 'data' run the trigger to the DAQ was provided by large pulses exceeding about $10 \mathrm{MeV}$ energy deposition in the detector. The rate of recorded events in the data run was a few events/hour consisting of muons and a tail of gamma events seen at higher energies due to their location close to PMTs. The $5 \mu$ s and $195 \mu$ s time periods before and after the trigger pulse, respectively, were digitised allowing for the delayed pulse from neutron capture to be recorded. Only pulses that exceeded $0.02 \mathrm{~V}$ in amplitude (equivalent to approximately $200 \mathrm{keV}$ energy deposition) were selected for further analysis. The presence of the afterpulses made 
impossible any analysis of neutron captures that could occur within the first $15-20 \mu$ s after a muon. Only pulses with the time delays from $40 \mu$ s to $195 \mu$ s were included in the final analysis.

Event waveforms were parameterised using the data reduction code similar to that written for the ZEPLIN-II experiment [3]. For each pulse on the waveform, the area (proportional to the charge and, hence, deposited energy), amplitude, width, mean time, full width at half maximum (FWHM) and arrival time were recorded.

Energy calibration of the scintillator was carried out with ${ }^{60} \mathrm{Co}$ gamma-ray source. Additional (even more accurate) calibration was provided by the peak in the energy spectrum of delayed pulses that corresponded to the Compton edge and full absorption of the $2.22 \mathrm{MeV}$ gamma-rays from neutron capture by hydrogen. Detector was also exposed to neutrons from Am-Be source. The good agreement observed between the measured and simulated time delay distributions proves the reliability of the model for neutron interactions and capture at $\mathrm{MeV}$ and sub-MeV energies.

The data on muon-induced neutrons were collected from August 2006 until April 2007. The total live time of the experiment was 204.8 days. Muon events were selected as those with high energy in the trigger pulse (see Ref. [5] for more details). Secondary, delayed, pulses were selected if their energy exceeded $0.55 \mathrm{MeV}$. During the experiment 10832 muons were detected translating to a rate of $52.9 \pm 0.5$ per day. Comparing the measured rate with the Monte Carlo predictions gives the total muon flux at Boulby as $(3.79 \pm 0.04$ (stat) \pm 0.11 (syst) $) \times 10^{-8} \mathrm{~cm}^{-2} \mathrm{~s}^{-1}$.

\section{Results}

The measured time delay distribution of delayed pulses in the events relative to trigger (muon) pulses agree well with the simulations [5]. The total number of secondary (delayed) pulses with energy deposition greater than $0.55 \mathrm{MeV}$ in the time window of $40-190 \mu$ s after the muon trigger was measured as 1037 whereas the number of muon triggers was 10832 giving the rate of $0.096 \pm$ 0.003 pulses/muon.

The delayed pulses in muon-induced events consist of two components: (i) an exponential-like time distribution of neutron captures and (ii) flat time distribution of background pulses. Similar time distribution for gamma-induced events (low energy trigger) is flat proving that the delayed pulses in gamma events are due to rare random coincidences. Gamma-induced events allow us to determine the flat background component or the rate of background pulses in muon events. The number of background events in 40-190 $\mu$ s time window after the gamma trigger is 351 for 21461 gamma triggers giving a rate of background pulses of $0.0164 \pm 0.0009$ per event. So the true rate of neutron pulses in muon events is the difference between the total rate of secondary pulses in these events and the rate of pulses due to random background coincidences (flat component). Hence the neutron rate, defined as the average number of detected neutrons per detected muon, is obtained as $0.079 \pm 0.003$ neutrons/muon in $40-190 \mu$ s time window above $0.55 \mathrm{MeV}$ threshold for energy deposition. The error is purely statistical. Systematic uncertainty is mainly due to the uncertainty in the pulse area - to - energy conversion and will be added to the predicted neutron yield.

Fig. 1 shows measured and simulated multiplicity distributions of secondary pulses for muon events, namely the number of events times the number of delayed pulses in the event as a function of the number of delayed pulses. Large neutron multiplicity is expected in some muon events due to enhanced neutron production in lead, proving the neutron origin of delayed pulses. 


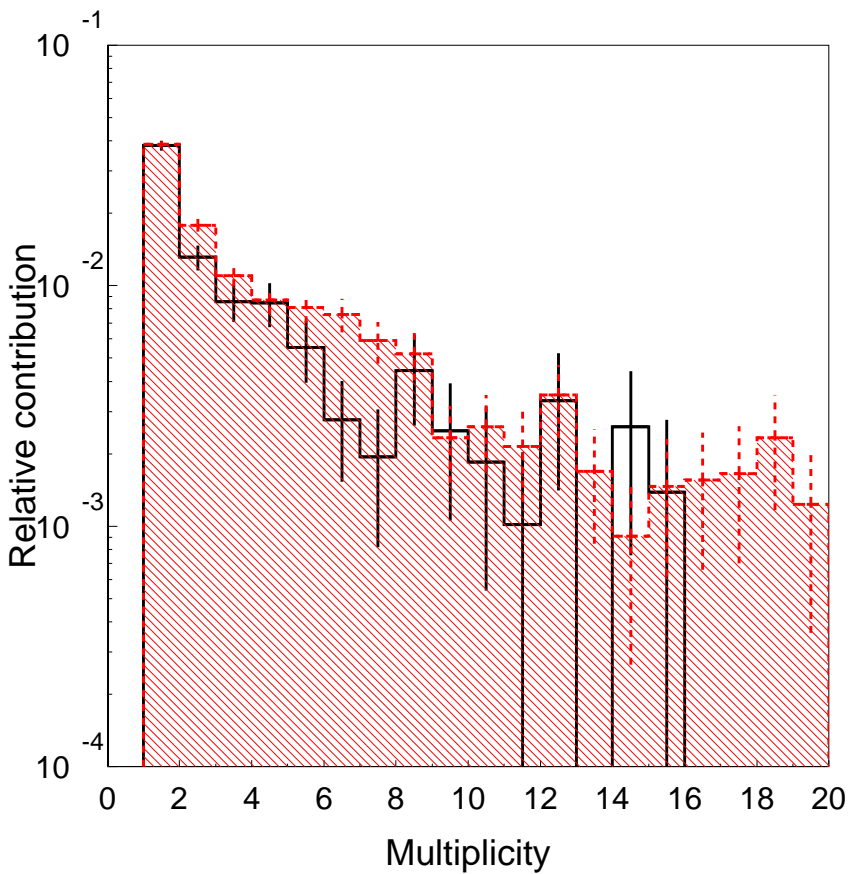

Figure 1: Measured (solid histogram) and simulated (dashed histogram and shaded area) multiplicity distributions of secondary pulses in muon events. First multiplicity bin with non-zero event content corresponds to one secondary pulse in the events.

Neutron production, transport and detection were simulated using GEANT4 [4] version 8.2. The laboratory hall, lead and wax shielding, veto and ZEPLIN-II detector were included in the simulations [6]. The physics models and particle production thresholds ('cuts') were essentially those described in Ref. [2], although other models were studied as described in Ref. [6]. Muons were sampled using the muon generator MUSUN [1] according to their energy spectrum and angular distribution at the Boulby Underground Laboratory. In total, about 120 million muons were sampled. This corresponded to a run time of about 960 days, 4.7 times that for real data. Comparison between multiplicity distributions for data and simulations is shown in Fig. 1. A smaller rate of events is observed for almost all neutron multiplicities compared to simulations.

The rate of simulated pulses from neutron capture gammas in 40-190 $\mu$ s can be directly compared to the measurements. This does not require any assumption about the shape of the time delay distribution other than a general agreement between measured and simulated distributions. The simulated rate of neutron capture gammas was found to be $0.143 \pm 0.002$ (stat.) \pm 0.009 (syst.) neutrons/muon above $0.55 \mathrm{MeV}$ energy deposition in 40-190 $\mu$ s time window. This value is 1.8 higher than the measured rate. Systematic uncertainty is mainly due to the $20 \%$ uncertainty in the energy scale. This was converted into the uncertainty of 0.009 neutrons/muon in the simulated neutron rate in the 40-190 $\mu$ s time window with a threshold of $0.55 \mathrm{MeV}$ for delayed pulses.

For practical use the measured neutron rate has to be converted to the neutron yield in a specific material, defined as the average number of neutrons produced by a muon along $1 \mathrm{~g} / \mathrm{cm}^{2}$ of its path. The most reliable and quasi model independent way of doing this is based on the following assumptions: 1) The geometry, neutron moderation and capture are described correctly in GEANT4; this is proven by the time delay distributions of data and calibration neutrons and by the agreement 
between GEANT4 and MCNPX (see Ref. [7] for discussion) for MeV and sub-MeV neutron transport; 2) Most neutrons (about 90\%) detected in our scintillator are produced in lead - this follows from simulations; 3) The ratio of measured-to-simulated neutron rates per detected muon is thus the same as for raw neutron yields in lead. Using the fact that the measured neutron rate is 1.8 times smaller than GEANT4 predictions, and the calculated neutron yield in the same model in lead is $2.37 \times 10^{-3}$ neutrons $/ \mathrm{muon} /\left(\mathrm{g} / \mathrm{cm}^{2}\right)$ for a muon energy of $260 \mathrm{GeV}$, we found the yield in lead, reconstructed from our measurements, to be $(1.31 \pm 0.06) \times 10^{-3}$ neutrons $/ \mathrm{muon} /\left(\mathrm{g} / \mathrm{cm}^{2}\right)$, for a mean muon energy of about $260 \mathrm{GeV}$.

The hypothetical contribution of a muon-induced neutron background for dark matter searches was checked using data from the ZEPLIN-II science run [3] with $225 \mathrm{~kg} \times$ days exposure. ZEPLINII was operated in coincidence with the active veto system described here. The data did not contain any neutron-induced nuclear recoil event in the target (ZEPLIN-II) in coincidence with a large muon signal in the active veto showing that for detectors with a few kilograms of target mass this background is not a severe threat at a depth of about $3 \mathrm{~km} \mathrm{w}$. e.

\section{Conclusions}

The muon-induced neutron rate at the Boulby Underground Laboratory was measured as $0.079 \pm 0.003$ (stat.) neutrons/muon using neutron-capture signals above $0.55 \mathrm{MeV}$ in a time window of 40-190 $\mu \mathrm{s}$ after the muon trigger. Accurate Monte Carlo simulations of the neutron production, transport and detection in a precisely modeled laboratory and experimental setup carried out using the GEANT4 toolkit, gave a rate of $0.143 \pm 0.002$ (stat.) \pm 0.009 (syst.) neutrons/muon for the same selection criteria. The simulated result is 1.8 times higher than the measured value. As the vast majority of neutrons detected in the current setup were produced in lead we evaluated from our measurements the total neutron yield in lead as $(1.31 \pm 0.06) \times 10^{-3}$ neutrons $/ \mathrm{muon} /\left(\mathrm{g} / \mathrm{cm}^{2}\right)$ for mean muon energy of about $260 \mathrm{GeV}$.

\section{Acknowledgements}

This work has been supported by the ILIAS integrating activity (Contract No. RII3-CT-2004506222) as part of the EU FP6 programme in Astroparticle Physics. We acknowledge the financial support from the STFC (UK), DoE (US), NSF (US) and FCT (Portugal). We also wish to thank Cleveland Potash Ltd for assistance.

\section{References}

[1] V. A. Kudryavtsev et al. Nucl. Instrum. \& Meth. in Phys. Res. A, 505 (2003) 688.

[2] H. M. Araújo et al. Nucl. Instrum. \& Meth. in Phys. Res. A, 545 (2005) 398.

[3] G. J. Alner et al. (ZEPLIN-II Collaboration). Astroparticle Phys., 28 (2007) 287.

[4] S. Agostinelli et al. Nucl. Instrum. \& Meth. in Phys. Res. A, 506 (2003) 250.

[5] H. M. Araújo et al. Astroparticle Phys., 29 (2008) 471.

[6] A. Lindote et al., submitted to Astroparticle Phys.; arXiv:0810.1682v1 [hep-ex].

[7] R. Lemrani et al. Nucl. Instrum. \& Meth. in Phys. Res. A, 560 (2006) 454. 